\title{
An Academic Hospitalist Model to Improve Healthcare Worker Communication and Learner Education: Results From a Quasi-experimental Study at a Veterans Affairs Medical Center
}

\author{
Sanjay Saint, MD, MPH ${ }^{1,2,3 *}$, Karen E. Fowler, MPH ${ }^{1,2}$, Sarah L. Krein, PhD, RN ${ }^{1,2,3}$, Scott A. Flanders, MD $^{3}$, \\ Timothy W. Bodnar, $\mathrm{MD}^{3}$, Eric Young, $\mathrm{MD}^{1,2}$, Richard H. Moseley, MD ${ }^{1,2,3}$
}

\begin{abstract}
'VA Ann Arbor Healthcare System, Ann Arbor, Michigan; 'VA Ann Arbor Hospital Outcomes Program of Excellence (HOPE) Initiative, Ann Arbor, Michigan; ${ }^{3}$ Department of Internal Medicine, University of Michigan Medical School, Ann Arbor, Michigan.
\end{abstract}

BACKGROUND: Although hospitalists may improve efficiency and quality of inpatient care, their effect on healthcare-worker communication and education has been less well-studied.

OBJECTIVE: To test various approaches to improving healthcare-worker communication and learner education within the context of a newly designed academic hospital medicine program.

DESIGN: Before-and-after design with concurrent control group.

SETTING: A Midwestern Veterans Affairs medical center.

INTERVENTION: Multimodal systems redesign of 1 of 4 medical teams (Gold team) that included clinical modifications (change in rounding structure, with inclusion of nurses, a Clinical Care Coordinator, and a pharmacist) and educational interventions (providing explicit expectations of learners and providing a reading list for both learners and attending physicians).

MEASUREMENTS: Number of admissions, length of stay, readmissions, house officer and medical student ratings of attendings' teaching, medical student internal medicine
National Board of Medical Examiners Subject Examination ("shelf" exam) scores, and clinical staff surveys.

RESULTS: Length of stay was reduced by about 0.3 days on all teams after the initiative began $(P=0.004)$, with no significant differences between Gold and non-Gold teams. The majority of physicians (83\%) and nurses (68\%) felt that including nurses during rounds improved healthcare-worker communication; significantly more nurses were satisfied with communication with the Gold team than with the other teams $(71 \%$ vs $53 \% ; P=0.02)$. Gold attendings generally received higher teaching scores compared with non-Gold attendings, and third-year medical students on the Gold team scored significantly higher on the shelf exam compared with non-Gold team students ( 84 vs $82 ; P=0.006$ ).

CONCLUSIONS: Academic hospitalists working within a systems redesign intervention were able to improve healthcare-worker communication and enhance learner education without increasing patient length of stay or readmission rates. Journal of Hospital Medicine 2013;8: 702-710. 2013 The Authors. Journal of Hospital Medicine published by Wiley Periodicals, Inc. on behalf of Society of Hospital Medicine
Improving quality while reducing costs remains important for hospitals across the United States, including the approximately 150 hospitals that are part of the Veterans Affairs (VA) healthcare system., The field of hospital medicine has grown rapidly, leading to predictions that the majority of inpatient care in the United States eventually will be delivered

*Address for correspondence and reprint requests: Sanjay Saint, MD, $\mathrm{MPH}$, VA Ann Arbor Healthcare System and University of Michigan Medical School, 2800 Plymouth Road, Building 16, Room 430W, Ann Arbor, Ml 48109; Telephone: 734-615-8341; Fax: 734-936-8944; E-mail: saint@med.umich.edu

Additional Supporting Information may be found in the online version of this article.

Received: August 8, 2013; Revised: October 1, 2013; Accepted:

October 2, 2013

Published 2013. This article is a U.S. Government work and is in the public domain in the USA. Journal of Hospital Medicine published by Wiley Periodicals, Inc. on behalf of Society of Hospital Medicine DOI 10.1002/jhm.2105

This is an open access article under the terms of the Creative Commons Attribution NonCommercial License, which permits use, distribution and reproduction in any medium, provided the original work is properly cited and is not used for commercial purposes.

Published online in Wiley Online Library (Wileyonlinelibrary.com). by hospitalists. ${ }^{3,4}$ In $2010,57 \%$ of US hospitals had hospitalists on staff, including $87 \%$ of hospitals with $\geq 200$ beds, ${ }^{5}$ and nearly $80 \%$ of VA hospitals. ${ }^{6}$

The demand for hospitalists within teaching hospitals has grown in part as a response to the mandate to reduce residency work hours. ${ }^{7}$ Furthermore, previous research has found that hospitalist care is associated with modest reductions in length of stay (LOS) and weak but inconsistent differences in quality. ${ }^{8}$ The educational effect of hospitalists has been far less examined. The limited number of studies published to date suggests that hospitalists may improve resident learning and house-officer satisfaction in academic medical centers and community teaching hospitals ${ }^{9-11}$ and provide positive experiences for medical students ${ }^{12,13}$; however, Wachter et al reported no significant changes in clinical outcomes or patient, faculty, and house-staff satisfaction in a newly designed hospital medicine service in San Francisco. ${ }^{14}$ Additionally, whether using hospitalists influences nurse-physician communication ${ }^{15}$ is unknown.

Recognizing the limited and sometimes conflicting evidence about the hospitalist model, we report the 
results of a 3-year quasi-experimental evaluation of the experience at our medical center with academic hospitalists. As part of a VA Systems Redesign Improvement Capability Grant-known as the Hospital Outcomes Program of Excellence (HOPE) Initiative-we created a hospitalist-based medicine team focused on quality improvement, medical education, and patient outcomes.

\section{METHODS}

\section{Setting and Design}

The main hospital of the VA Ann Arbor Healthcare System, located in Ann Arbor, Michigan, operates 105 acute-care beds and 40 extended-care beds. At the time of this evaluation, the medicine service consisted of 4 internal medicine teams-Gold, Silver, Burgundy, and Yellow-each of which was responsible for admitting patients on a rotating basis every fourth day, with limited numbers of admissions occurring between each team's primary admitting day. Each team is led by an attending physician, a boardcertified (or board-eligible) general internist or subspecialist who is also a faculty member at the University of Michigan Medical School. Each team has a senior medical resident, 2 to 3 interns, and 3 to 5 medical students (mostly third-year students). In total, there are approximately 50 senior medical residents, 60 interns, and 170 medical students who rotate through the medicine service each year. Traditional rounding involves the medical students and interns receiving sign-out from the overnight team in the morning, then "pre-rounding" on each patient by obtaining an interval history, performing an exam, and checking any test results. A tentative plan of care is formed with the senior medical resident, usually by discussing each patient very quickly in the team room. Attending rounds are then conducted, with the physician team visiting each patient one by one to review and plan all aspects of care in detail. When time allows, small segments of teaching may occur during these "attending work rounds." This system had been in place for $>20$ years.

Resulting in part from a grant received from the VA Systems Redesign Central Office (ie, the HOPE Initiative), the Gold team was modified in July 2009 and an academic hospitalist (S.S.) was assigned to head this team. Specific hospitalists were selected by the Associate Chief of Medicine (S.S.) and the Chief of Medicine (R.H.M.) to serve as Gold team attendings on a regular basis. The other teams continued to be overseen by the Chief of Medicine, and the Gold team remained within the medicine service. Characteristics of the Gold and non-Gold team attendings can be found in Table 1 . The 3 other teams initially were noninterventional concurrent control groups. However, during the second year of the evaluation, the Silver team adopted some of the initiatives as a result of the preliminary findings observed on Gold. Specifically, in the second year of the evaluation, approximately $42 \%$ of attend-
TABLE 1. Characteristics of Gold Team and Non-Gold Team Attendings Postinitiative (July 2009-June 2012)

\begin{tabular}{lcc}
\hline Characteristic & Gold Team & Non-Gold Teams \\
\hline Total number of attendings & 14 & 57 \\
Sex, \% & 79 & 58 \\
$\quad$ Male & 21 & 42 \\
Female & $10(1-30)$ & $7(1-41)$ \\
Median years postresidency (range) & 14 & 40 \\
Subspecialists, \% & $30(5-92)$ \\
Median days on service per year (range) & $53(5-74)$ & \\
\hline
\end{tabular}

TABLE 2. Description of Gold Team Interventions

\begin{tabular}{ll}
\hline Clinical Interventions & \multicolumn{1}{c}{ Educational Interventions } \\
\hline Modified structure of attending rounds & Modified structure of attending rounds \\
Circle of Concern rounds & Attending reading list \\
Clinical Care Coordinator & "Nifty Fifty" reading list for learners \\
Regular attending team meetings & Website to provide expectations to learners \\
Two-month per year commitment by attendings & \\
\hline
\end{tabular}

ings on the Silver team were from the Gold team. This increased in the third year to $67 \%$ of coverage by Gold team attendings on the Silver team. The evaluation of the Gold team ended in June 2012.

The clinical interventions implemented on the Gold team were quality-improvement work and were therefore exempt from institutional review board review. Human subjects' approval was, however, received to conduct interviews as part of a qualitative assessment.

\section{Clinical Interventions}

Several interventions involving the clinical care delivered were introduced on the Gold team, with a focus on improving communication among healthcare workers (Table 2).

\section{Structure of Attending Rounds}

The structure of morning rounds was modified on the Gold team. Similar to the traditional structure, medical students and interns on the Gold team receive sign-out from the overnight team in the morning. However, interns and students may or may not conduct "pre-rounds" on each patient. The majority of time between sign-out and the arrival of the attending physician is spent on "work rounds." The senior resident leads rounds with the interns and students, discussing each patient while focusing on overnight events and current symptoms, new physicalexamination findings, and laboratory and test data. The plan of care to be presented to the attending is then formulated with the senior resident. The attending physician then leads "Circle of Concern" rounds with an expanded team, including a charge nurse, a clinical pharmacist, and a nurse Clinical Care Coordinator. Attending rounds tend to use an "E-AP" format: significant Events overnight are discussed, 
TABLE 3. Reading List for Attending Physicians

\begin{tabular}{ll}
\hline Book Title & \multicolumn{1}{c}{ Author(s) } \\
\hline The One Minute Manager & Ken Blanchard and Spencer Johnson \\
Good to Great & Jim Collins \\
Good to Great and the Social Sectors & Jim Collins \\
The Checklist Manifesto: How to Get Things Right & Atul Gawande \\
The Five Dysfunctions of a Team: A Leadership Fable & Patrick Lencioni \\
Getting to Yes: Negotiating Agreement Without Giving In & Roger Fisher, William Ury, and Bruce Patton \\
The Effective Executive: The Definitive Guide to Getting the Right Things Done & Peter Drucker \\
A Sense of Urgency & John Kotter \\
The Power of Positive Deviance: How Unlikely Innovators Solve the World's Toughest Problems & Richard Pascale, Jerry Sternin, and Monique Sternin \\
On the Mend: Revolutionizing Healthcare to Save Lives and Transform the Industry & John Toussaint and Roger Gerard \\
Outtliers: The Story of Success & Malcolm Gladwell \\
Nursing Against the Odds: How Health Care Cost Cutting, Media Stereotypes, and Medical Hubris Undermine Nurses and Patient Care & Suzanne Gordon \\
How the Mighty Fall and Why Some Companies Never Give In & Jim Collins \\
What the Best College Teachers Do & Ken Bain \\
The Creative Destruction of Medicine & Eric Topol \\
What Got You Here Won't Get You There: How Successful People Become Even More Successful! & Marshall Goldsmith \\
\hline
\end{tabular}

followed by an Assessment \& Plan by problem for the top active problems. Using this model, the attendings are able to focus more on teaching and discussing the patient plan than in the traditional model (in which the learner presents the details of the subjective, objective, laboratory, and radiographic data, with limited time left for the assessment and plan for each problem).

\section{Circle of Concern Rounds}

Suzanne Gordon described the Circle of Concern in her book Nursing Against the Odds. ${ }^{16}$ From her observations, she noted that physicians typically form a circle to discuss patient care during rounds. The circle expands when another physician joins the group; however, the circle does not similarly expand to include nurses when they approach the group. Instead, nurses typically remain on the periphery, listening silently or trying to communicate to physicians' backs. ${ }^{16}$ Thus, to promote nurse-physician communication, Circle of Concern rounds were formally introduced on the Gold team. Each morning, the charge nurse rounds with the team and is encouraged to bring up nursing concerns. The inpatient clinical pharmacist is also included 2 to 3 times per week to help provide education to residents and students and perform medication reconciliation.

\section{Clinical Care Coordinator}

The role of the nurse Clinical Care Coordinator-also introduced on the Gold team-is to provide continuity of patient care, facilitate interdisciplinary communication, facilitate patient discharge, ensure appropriate appointments are scheduled, communicate with the ambulatory care service to ensure proper transition between inpatient and outpatient care, and help educate residents and students on VA procedures and resources.

\section{Regular Gold Team Meetings}

All Gold team attendings are expected to dedicate 2 months per year to inpatient service (divided into half-month blocks), instead of the average 1 month per year for attendings on the other teams. The Gold team attendings, unlike the other teams, also attend bimonthly meetings to discuss strategies for running the team.

\section{Educational Interventions}

Given the high number of learners on the medicine service, we wanted to enhance the educational experience for our learners. We thus implemented various interventions, in addition to the change in the structure of rounds, as described below.

\section{Reading List for Learners: The "Nifty Fifty"}

Because reading about clinical medicine is an integral part of medical education, we make explicit our expectation that residents and students read something clinically relevant every day. To promote this, we have provided a "Nifty Fifty" reading list of key articles. The PDF of each article is provided, along with a brief summary highlighting key points.

\section{Reading List for Gold Attendings and Support Staff}

To promote a common understanding of leadership techniques, management books are provided to Gold attending physicians and other members of the team (eg, Care Coordinator, nurse researcher, systems redesign engineer). One book is discussed at each Gold team meeting (Table 3), with participants taking turns leading the discussion.

\section{Website}

A HOPE Initiative website was created (http:// www.va-hope.org) to help introduce residents and students to the Gold team. The website includes key 
resources, such as the "Nifty Fifty" reading list and "The Seven Suggestions" orientation sheet so they know what to expect while they are on service.

\section{Qualitative Assessment}

To evaluate our efforts, we conducted a thorough qualitative assessment during the third year of the program. A total of 35 semistructured qualitative interviews were conducted with patients and staff from all levels of the organization, including senior leadership. The qualitative assessment was led by research staff from the Center for Clinical Management Research, who were minimally involved in the redesign effort and could provide an unbiased view of the initiative. Field notes from the semistructured interviews were analyzed, with themes developed using a descriptive approach and through discussion by a multidisciplinary team, which included building team consensus on findings that were supported by clear evidence in the data. ${ }^{17}$

\section{Quantitative Outcome Measures Clinical Outcomes}

To determine if our communication and educational interventions had an impact on patient care, we used hospital administrative data to evaluate admission rates, LOS, and readmission rates for all 4 of the medicine teams. Additional clinical measures were assessed as needed. For example, we monitored the impact of the clinical pharmacist during a 4-week pilot study by asking the Clinical Care Coordinator to track the proportion of patient encounters $(n=170)$ in which the clinical pharmacist changed management or provided education to team members. Additionally, 2 staff surveys were conducted. The first survey focused on healthcare-worker communication and was given to inpatient nurses and physicians (including attendings, residents, and medical students) who were recently on an inpatient medical service rotation. The survey included questions from previously validated communication measures, ${ }^{18-20}$ as well as study-specific questions. The second survey evaluated the new role of the Clinical Care Coordinator (Appendix). Both physicians and nurses who interacted with the Gold team's Clinical Care Coordinator were asked to complete this survey.

\section{Educational Outcomes}

To assess the educational interventions, we used learner evaluations of attendings, by both residents and medical students, and standardized internal medicine National Board of Medical Examiners Subject Examination (or "shelf") scores for third-year medical students. A separate evaluation of medical student perceptions of the rounding structure introduced on the Gold team using survey design has already been published. $^{21}$

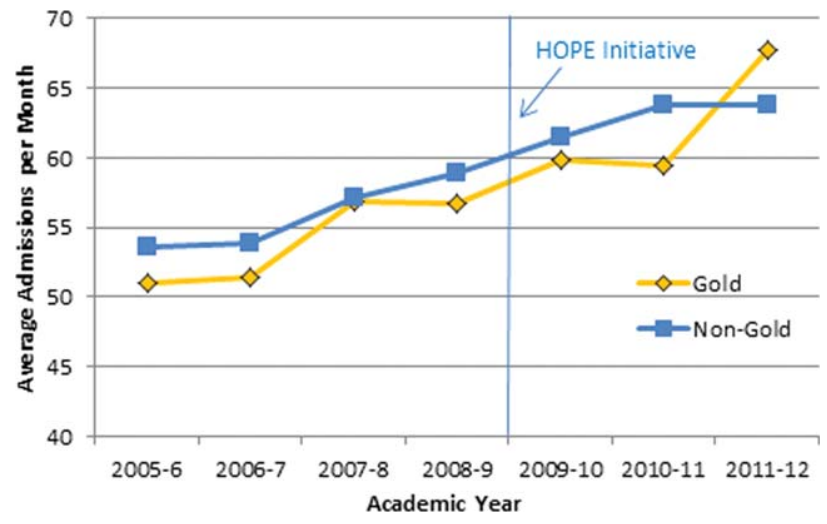

FIG. 1. Admissions per month. Abbreviations: HOPE, Hospital Outcomes Program of Excellence.

\section{Statistical Analyses}

Data from all sources were analyzed using SAS 9.3 (SAS Institute, Inc., Cary, NC). Outliers for the LOS variable were removed from the analysis. Means and frequency distributions were examined for all variables. Student $t$ tests and $\chi^{2}$ tests of independence were used to compare data between groups. Multivariable linear regression models controlling for time (preintervention vs postintervention) were used to assess the effect of the HOPE Initiative on patient LOS and readmission rates. In all cases, 2-tailed $P$ values of 0.05 or less were considered statistically significant.

\section{Role of the Funding Source}

The VA Office of Systems Redesign provided funding but was not involved in the design or conduct of the study, data analysis, or preparation of the manuscript.

\section{RESULTS \\ Clinical Outcomes \\ Patient Outcomes}

Our multivariable linear regression analysis, controlling for time, showed a significant reduction in LOS of approximately 0.3 days on all teams after the HOPE Initiative began $(P=0.004)$. There were no significant differences between the Gold and non-Gold teams in the multivariate models when controlling for time for any of the patient-outcome measures. The number of admissions increased for all 4 medical teams (Figure 1), but, as shown in Figures 2 and 3, the readmission rates for all teams remained relatively stable over this same period of time.

\section{Clinical Pharmacist on Gold Team Rounds}

The inpatient clinical pharmacist changed the management plan for $22 \%$ of the patients seen on rounds. Contributions from the clinical pharmacist included adjusting the dosing of ordered medication and correcting medication reconciliation. Education and pharmaceutical information was provided to the team in another $6 \%$ of the 170 consecutive patient encounters evaluated. 


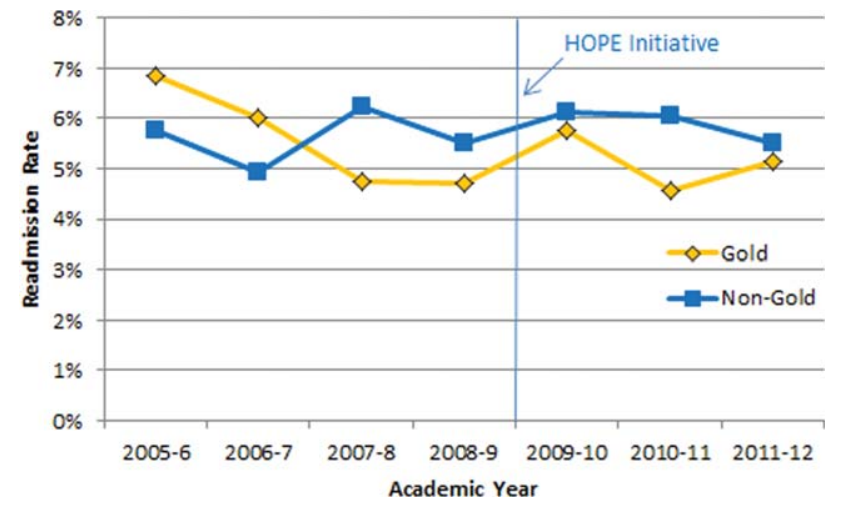

FIG. 2. Seven-day readmission rate. Abbreviations: HOPE, Hospital Outcomes Program of Excellence.

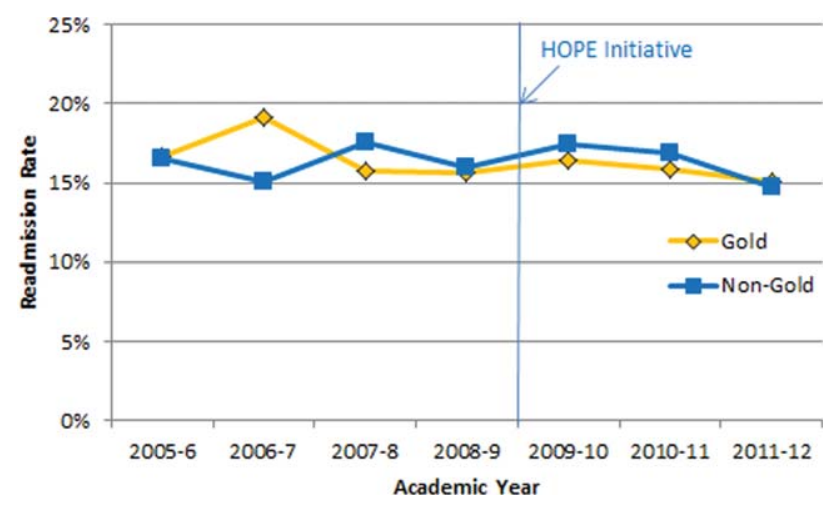

FIG. 3. Thirty-day readmission rate. Abbreviations: HOPE, Hospital Outcomes Program of Excellence.

\section{Perception of Circle of Concern Rounds}

Circle of Concern rounds were generally well-received by both nurses and physicians. In a healthcare-worker communication survey, completed by 38 physicians (62\% response rate) and 48 nurses $(54 \%$ response rate), the majority of both physicians $(83 \%)$ and nurses $(68 \%)$ felt Circle of Concern rounds improved communication.

\section{Nurse Perception of Communication}

The healthcare-worker communication survey asked inpatient nurses to rate communication between nurses and physicians on each of the 4 medicine teams. Significantly more nurses were satisfied with communication with the Gold team (71\%) compared with the other 3 medicine teams $(53 \% ; P=0.02)$ (Figure 4).

\section{Perception of the Clinical Care Coordinator}

In total, 20 physicians ( $87 \%$ response rate) and 10 nurses $(56 \%$ response rate) completed the Clinical Care Coordinator survey. The physician results were overwhelmingly positive: $100 \%$ were satisfied or very satisfied with the role; $100 \%$ felt each team should have a Clinical Care Coordinator; and 100\% agreed or strongly agreed that the Clinical Care Coordinator ensures that appropriate follow-up is arranged, provides continuity of care, assists with interdisciplinary

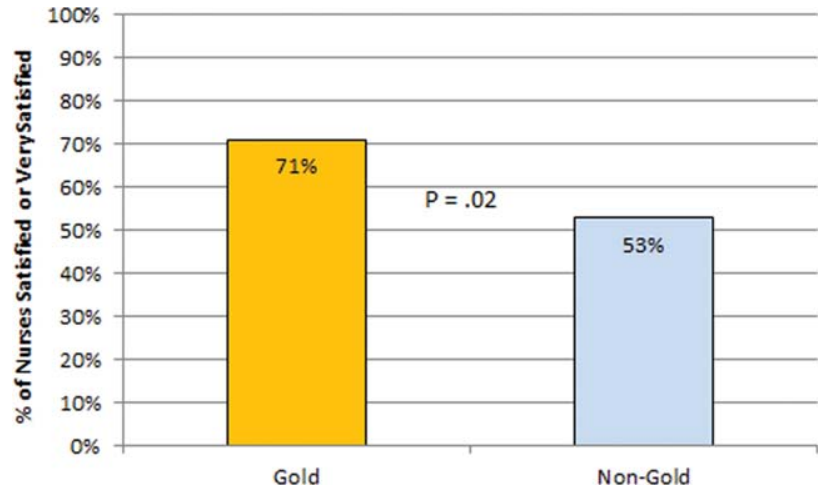

FIG. 4. Nurse satisfaction with communication on team.

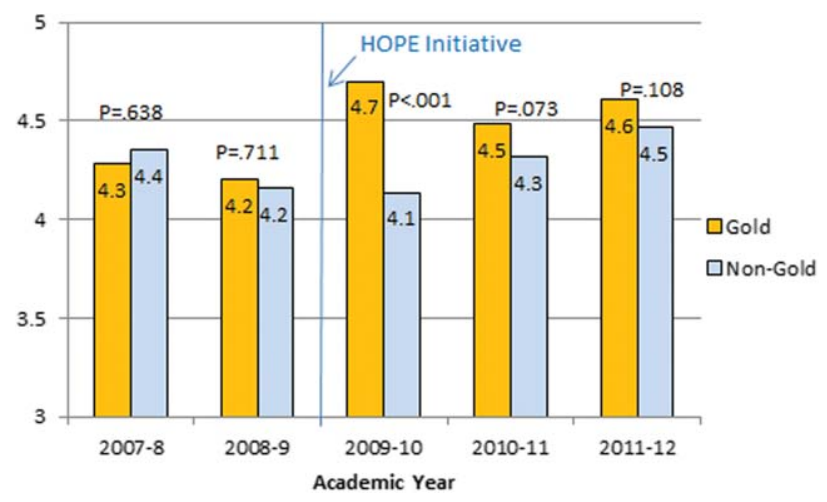

FIG. 5. House officer rating of attendings (1= unsatisfactory, $5=$ outstanding). Abbreviations: HOPE, Hospital Outcomes Program of Excellence.

communication, and helps facilitate discharge. The majority of nurses was also satisfied or very satisfied with the Clinical Care Coordinator role and felt each team should have one.

\section{Educational Outcomes House Officer Evaluation of Attendings}

Monthly evaluations of attending physicians by house officers (Figure 5) revealed that prior to the HOPE Initiative, little differences were observed between teams, as would be expected because attending assignment was largely random. After the intervention date of July 2009, however, significant differences were noted, with Gold team attendings receiving significantly higher teaching evaluations immediately after the introduction of the HOPE Initiative. Although ratings for Gold attendings remained more favorable, the difference was no longer statistically significant in the second and third year of the initiative, likely due to Gold attendings serving on other medicine teams, which contributed to an improvement in ratings of all attendings.

\section{Medical Student Evaluation of Attendings}

Monthly evaluations of attending physicians by thirdyear medical students (Figure 6) revealed differences 


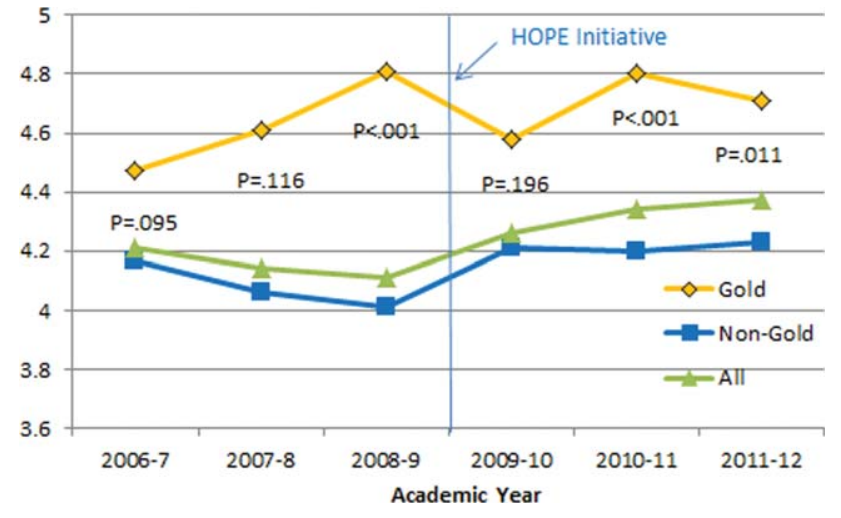

FIG. 6. Medical student rating of overall quality of teaching of attending ( $1=$ poor, 5 = excellent). Abbreviations: HOPE, Hospital Outcomes Program of Excellence.

between the Gold attendings and all others, with the attendings that joined the Gold team in 2009 receiving higher teaching evaluations even before the HOPE Initiative started. However, this difference remained statistically significant in years 2 and 3 postinitiative, despite the addition of 4 new junior attendings.

\section{Medical Student Medicine Shelf Scores}

The national average on the "shelf" exam, which reflects learning after the internal medicine third-year clerkship, has ranged from 75 to 78 for the past several years, with University of Michigan students averaging significantly higher scores prior to and after the HOPE Initiative. However, following the HOPE Initiative, third-year medical students on the Gold team scored significantly higher on the shelf exam compared with their colleagues on the non-Gold teams (84 vs $82 ; P=0.006$ ). This difference in the shelf exam scores, although small, is statistically significant. It represents a measurable improvement in shelf scores in our system and demonstrates the potential educational benefit for the students. Over this same time period, scores on the United States Medical Licensing Exam, given to medical students at the beginning of their third year, remained stable (233 pre-HOPE Initiative; 234 post-HOPE Initiative).

\section{Qualitative Assessment}

Qualitative data collected as part of our evaluation of the HOPE Initiative also suggested that nursephysician communication had improved since the start of the project. In particular, they reported positively on the Gold team in general, the Circle of Concern rounds, and the Clinical Care Coordinator (Table 4).

\section{DISCUSSION}

Within academic medical centers, hospitalists are expected to care for patients, teach, and help improve the quality and efficiency of hospital-based care. ${ }^{7}$ The Department of Veterans Affairs runs the largest integrated healthcare system in the United States, with

\begin{tabular}{|c|c|}
\hline Staff Type & Statement* \\
\hline Nurse & $\begin{array}{l}\text { [Gold is] above and beyond other [teams]. Other teams don't run as } \\
\text { smoothly. }\end{array}$ \\
\hline Nurse & $\begin{array}{l}\text { There has been a difference in communication [on Gold]. You can tell the } \\
\text { difference in how they communicate with staff. We know the Clinical } \\
\text { Care Coordinator or charge nurse is rounding with that team, so there } \\
\text { is more communication. }\end{array}$ \\
\hline Nurse & $\begin{array}{l}\text { The most important thing that has improved communication is the Circle } \\
\text { of Concern rounds. }\end{array}$ \\
\hline Physician & $\begin{array}{l}\text { The Gold Clinical Care Coordinator] expedites care, not only what to do } \\
\text { but who to call. She can convey the urgency. On rounds she is able to } \\
\text { break off, put in an order, place a call, talk to a patient. Things that } \\
\text { we would do at } 11 \text { AM she gets to at } 9 \text { AM. A couple of hours may not } \\
\text { seem like much, but sometimes it can make the difference between } \\
\text { things happening that day instead of the next. }\end{array}$ \\
\hline Physician & $\begin{array}{l}\text { The Clinical Care Coordinator is completely indispensable. Major benefit to } \\
\text { providing care to Veterans. }\end{array}$ \\
\hline Physician & I like to think Gold has lifted all of the teams to a higher level. \\
\hline Medical student & $\begin{array}{l}\text { It may be due to personalities vs the Gold [team] itself, but there is more } \\
\text { emphasis on best practices. Are we following guidelines even if it is } \\
\text { not related to the primary reason for admission? }\end{array}$ \\
\hline Medical student & $\begin{array}{l}\text { Gold is very collegial and nurses/physicians know one another by name. } \\
\text { Physicians request rather than order; this sets a good example to me } \\
\text { on how to approach the nurses. }\end{array}$ \\
\hline Chief resident & $\begin{array}{l}\text { [Gold attendings] encourage senior residents to take charge and run the } \\
\text { team, although the attending is there for back-up and support. This } \\
\text { provides great learning for the residents. Interns and medical students } \\
\text { also are affected because they have to step up their game as well. }\end{array}$ \\
\hline
\end{tabular}

NOTE: *Statements represent thoughts suggested by the interviewees as recorded in the interview notes. These statements may be paraphrased and are not necessarily verbatim quotations.

approximately $80 \%$ of VA hospitals having hospital medicine programs. Overall, one-third of US residents perform part of their residency training at a VA hospital. $^{22,23}$ Thus, the effects of a system-wide change at a VA hospital may have implications throughout the country. We studied one such intervention. Our primary findings are that we were able to improve communication and learner education with minimal effects on patient outcomes. While overall LOS decreased slightly postintervention, after taking into account secular trends, readmission rates did not.

We are not the first to evaluate a hospital medicine team using a quasi-experimental design. For example, Meltzer and colleagues evaluated a hospitalist program at the University of Chicago Medical Center and found that, by the second year of operation, hospitalist care was associated with significantly shorter LOS (0.49 days), reduced costs, and decreased mortality. ${ }^{24}$ Auerbach also evaluated a newly created hospital medicine service, finding decreased LOS (0.61 days), lower costs, and lower risk of mortality by the second year of the program. ${ }^{25}$

Improving nurse-physician communication is considered important for avoiding medical error, ${ }^{26}$ yet there has been limited empirical study of methods to improve communication within the medical profession. ${ }^{27}$ Based both on our surveys and qualitative interviews, healthcare-worker communication 
appeared to improve on the Gold team during the study. A key component of this improvement is likely related to instituting Circle of Concern rounds, in which nurses joined the medical team during attending rounds. Such an intervention likely helped to address "organizational silence" 28 and enhance the psychological safety of the nursing staff, because the attending physician was proactive about soliciting the input of nurses during rounds. ${ }^{29}$ Such leader inclusiveness- "words and deeds exhibited by leaders that invite and appreciate others' contributions"-can aid interdisciplinary teams in overcoming the negative effects of status differences, thereby promoting collaboration. ${ }^{29}$ The inclusion of nurses on rounds is also relationship-building, which Gotlib Conn and colleagues found was important to improved interprofessional communication and collaboration. ${ }^{30}$ In the future, using a tool such as the Teamwork Effectiveness Assessment Module (TEAM) developed by the American Board of Internal Medicine ${ }^{31}$ could provide further evaluation of the impact on interprofessional teamwork and communication.

The focus on learner education, though evaluated in prior studies, is also novel. One previous survey of medical students showed that engaging students in substantive discussions is associated with greater student satisfaction. ${ }^{32}$ Another survey of medical students found that attendings who were enthusiastic about teaching, inspired confidence in knowledge and skills, provided useful feedback, and encouraged increased student responsibility were viewed as more effective teachers. ${ }^{33}$ No previous study that we are aware of, however, has looked at actual educational outcomes, such as shelf scores. The National Board of Medical Examiners reports that the Medicine subject exam is "scaled to have a mean of 70 and a standard deviation of $8 . "{ }^{34}$ Thus, a mean increase in score of 2 points is small, but not trivial. This shows improvement in a "hard" educational outcome. Additionally, 2 points, although small in the context of total score and standard deviation, may make a substantial difference to an individual student in terms of overall grade, and, thus, residency applications. Our finding that third-year medical students on the Gold team performed significantly better than University of Michigan third-year medical students on other teams is an intriguing finding that warrants confirmation. On the other hand, this finding is consistent with a previous report evaluating learner satisfaction in which Bodnar et al found improved ratings of quantity and quality of teaching on teams with a nontraditional structure (Gold team). ${ }^{21}$ Moreover, despite relatively few studies, the reason underlying the educational benefit of hospitalists should surprise few. The hospitalist model ensures that learners are supervised by physicians who are experts in the care of hospitalized patients. ${ }^{35}$ Hospitalists hired at teaching hospitals to work on services with learners are generally chosen because they possess superior educational skills. ${ }^{7}$

Our findings should be interpreted in the context of the following limitations. First, our study focused on a single academically affiliated VA hospital. As other VA hospitals are pursuing a similar approach (eg, the Houston and Detroit VA medical centers), replicating our results will be important. Second, the VA system, although the largest integrated healthcare system in the United States, has unique characteristics-such as an integrated electronic health record and predominantly male patient population-that may make generalizations to the larger US healthcare system challenging. Third, there was a slightly lower response rate among nurses on a few of the surveys to evaluate our efforts; however, this rate of response is standard at our facility. Finally, our evaluation lacks an empirical measure of healthcareworker communication, such as incident reports.

Despite these limitations, our results have important implications. Using both quantitative and qualitative assessment, we found that academic hospitalists have the ability to improve healthcare-worker communication and enhance learner education without increasing LOS. These findings are directly applicable to VA medical centers and potentially applicable to other academic medical centers.

\section{Acknowledgments}

The authors thank Milisa Manojlovich, PhD, RN, Edward Kennedy, MS, and Andrew Hickner, MSI, for help with preparation of this manuscript.

Disclosures: This work was funded by a US Department of Veterans Affairs, Office of Systems Redesign Improvement Capability grant. The findings and conclusions in this report are those of the authors and do not necessarily represent the position or policy of the Department of Veterans Affairs. Dr. Saint reports receiving travel reimbursement for giving invited talks at the Society of Hospital Medicine's National Meeting, as well as serving on the advisory boards of Doximity and Jvion. 


\section{APPENDIX}

\section{Survey to Evaluate the Care Coordinator Position}

\begin{tabular}{|c|c|c|c|c|c|c|}
\hline & Yes & & No & & & Not Sure \\
\hline Q1. Are you familiar with the role of the Care Coordinator on the Gold Service (Susan Lee)? & 1 & & 2 & & & 3 \\
\hline \multicolumn{7}{|l|}{ Please indicate how much you agree or disagree with the statements below. } \\
\hline & $\begin{array}{l}\text { Strongly } \\
\text { Agree }\end{array}$ & Agree & Neutral & Disagree & $\begin{array}{l}\text { Strongly } \\
\text { Disagree }\end{array}$ & $\begin{array}{l}\text { Don't } \\
\text { Know }\end{array}$ \\
\hline Q2. The Care Coordinator ensures that appropriate primary care follow-up and any other appropriate services are arranged. & 1 & 2 & 3 & 4 & 5 & 9 \\
\hline Q3. The Care Coordinator provides continuity of patient care on the Gold Service. & 1 & 2 & 3 & 4 & 5 & 9 \\
\hline Q4. The Care Coordinator helps educate House Officers and Medical Students on VA processes (e.g., CPRS). & 1 & 2 & 3 & 4 & 5 & 9 \\
\hline $\begin{array}{l}\text { Q5. The Care Coordinator assists with interdisciplinary communication between the medical team and other } \\
\text { services (e.g., nursing, ambulatory care, pharmacy, social work) }\end{array}$ & 1 & 2 & 3 & 4 & 5 & 9 \\
\hline Q6. The Care Coordinator helps facilitate patient discharge. & 1 & 2 & 3 & 4 & 5 & 9 \\
\hline Q7. The Care Coordinator initiates communication with the ambulatory care teams to coordinate care. & 1 & 2 & 3 & 4 & 5 & 9 \\
\hline
\end{tabular}

\begin{tabular}{|c|c|c|c|c|c|}
\hline & & & & Yes & No \\
\hline \multicolumn{4}{|c|}{ Q8. Are you a physician (attending or resident), or medical student who has been on more than one medical team at the VA (Gold, Silver, Burgundy, or Yellow)? } & 1 & 2 \\
\hline \multicolumn{6}{|c|}{$\begin{array}{l}\text { If no, please skip to } 013 \\
\text { If yes, comparing your experience on the Gold Service (with the Care Coordinator) to your experience on any of the other services (Silver, Burgundy, or Yellow): }\end{array}$} \\
\hline & Not at All & Very Little & Somewhat & & To a Great Extent \\
\hline Q9. To what extent does the presence of a Care Coordinator affect patient care? & 1 & 2 & 3 & & 4 \\
\hline Q10. To what extent does the presence of a Care Coordinator improve patient flow? & 1 & 2 & 3 & & 4 \\
\hline Q11. To what extent does the presence of a Care Coordinator assist with education? & 1 & 2 & 3 & & 4 \\
\hline Q12. To what extent does the presence of a Care Coordinator contribute to attending rounds? & 1 & 2 & 3 & & 4 \\
\hline
\end{tabular}

\begin{tabular}{lcc}
\hline & Yes & No \\
\hline Q13. Do you work [as a nurse] in ambulatory care? & 1 & 2 \\
\hline
\end{tabular}

If no, please skip to Q17.

Ifyes, comparing your experience with the Gold Service (with the Care Coordinator) to the other services (Silver, Burgundy, or Yellow):

\begin{tabular}{|c|c|c|c|c|}
\hline & Not at All & Very Little & Somewhat & To a Great Extent \\
\hline Q14. To what extent does the presence of a Care Coordinator improve coordination of care between inpatient and outpatient services? & 1 & 2 & 3 & 4 \\
\hline Q15. To what extent does the presence of a Care Coordinator help identify high risk patients who require follow-up? & 1 & 2 & 3 & 4 \\
\hline Q16. To what extent does the presence of a Care Coordinator ensure follow-up appointments are scheduled? & 1 & 2 & 3 & 4 \\
\hline Yes & & No & & Not Sure \\
\hline $\begin{array}{l}\text { Q17. Do you think each medical team should have a Care Coordinator? } \\
\text { Q18. Are there any additional tasks or duties you think would improve the effectiveness of the Care Coordinator? }\end{array}$ & & 2 & & 3 \\
\hline
\end{tabular}

\begin{tabular}{|c|c|c|c|c|c|}
\hline & Very Satisfied & Satisfied & Neutral & Dissatisfied & Very Dissatisfied \\
\hline $\begin{array}{l}\text { Q19. Overall how satisfied are you with the role of the Care Coordinator on the Gold Service? } \\
\text { Q20. Do you have any other comments about the role of the Care Coordinator? }\end{array}$ & 1 & 2 & 3 & 4 & 5 \\
\hline
\end{tabular}

\footnotetext{
Q21. What is your position?

1. Physician (attending or resident) or medical student

2. Nurse (inpatient or ambulatory care)
} 


\section{References}

1. Kohn LT, Corrigan JM, Donaldson MS, eds. To Err Is Human: Building a Safer Health System. Washington, D.C.: National Academies Press; 2000.

2. Institute of Medicine of the National Academies. Crossing the Quality Chasm: A New Health System for the 21st Century. Washington, D.C.: National Academies Press; 2001.

3. Kuo YF, Sharma G, Freeman JL, Goodwin JS. Growth in the care of older patients by hospitalists in the United States. N Engl J Med. 2009;360(11):1102-1112.

4. Wachter RM. Growth in care provided by hospitalists. N Engl J Med. 2009;360(26):2789-2791.

5. American Hospital Association. AHA Annual Survey of Hospitals, 2010. Chicago, IL: Health Forum, LLC; 2010.

6. Krein SL, Kowalski CP, Hofer TP, Saint S. Preventing hospitalacquired infections: a national survey of practices reported by U.S. hospitals in 2005 and 2009. J Gen Intern Med. 2012;27(7):773-779.

7. Saint S, Flanders SA. Hospitalists in teaching hospitals: opportunities but not without danger. I Gen Intern Med. 2004;19(4):392-393.

8. White HL, Glazier RH. Do hospitalist physicians improve the quality of inpatient care delivery? A systematic review of process, efficiency and outcome measures. BMC Med. 2011;9:58.

9. Natarajan P, Ranji SR, Auerbach AD, Hauer KE. Effect of hospitalist attending physicians on trainee educational experiences: a systematic review. J Hosp Med. 2009;4(8):490-498.

10. Chung P, Morrison J, Jin L, Levinson W, Humphrey H, Meltzer D. Resident satisfaction on an academic hospitalist service: time to teach. Am J Med. 2002;112(7):597-601.

11. Kulaga ME, Charney P, O'Mahony SP, et al. The positive impact of initiation of hospitalist clinician educators. J Gen Intern Med. 2004; 19(4):293-301.

12. Geskey JM, Kees-Folts D. Third-year medical students' evaluation of hospitalist and nonhospitalist faculty during the inpatient portion of their pediatrics clerkships. J Hosp Med. 2007;2(1):17-22.

13. Hunter AJ, Desai SS, Harrison RA, Chan BK. Medical student evaluation of the quality of hospitalist and nonhospitalist teaching faculty on inpatient medicine rotations. Acad Med. 2004;79(1):7882 .

14. Wachter RM, Katz P, Showstack J, Bindman AB, Goldman L. Reorganizing an academic medical service: impact on cost, quality, patient satisfaction, and education. JAMA. 1998;279(19):1560-1565.

15. Manojlovich M. Nurse/physician communication through a sensemaking lens: shifting the paradigm to improve patient safety. Med Care. 2010;48(11):941-946.

16. Gordon S. Nursing Against the Odds: How Health Care Cost Cutting, Media Stereotypes, and Medical Hubris Undermine Nurses and Patient Care. Ithaca, NY: Cornell University Press; 2005.

17. Sandelowski M. Focus on research methods: whatever happened to qualitative description? Res Nurs Health. 2000;23:334-340.

18. Shortell SM, Rousseau DM, Gillies RR, Devers KJ, Simons TL. Organizational assessment in intensive care units (ICUs): construct development, reliability, and validity of the ICU nurse-physician questionnaire. Med Care. 1991;29(8):709-726.
19. Baggs JG. Development of an instrument to measure collaboration and satisfaction about care decisions. J Adv Nurs. 1994;20(1):176-182.

20. Lake ET. Development of the practice environment scale of the Nursing Work Index. Res Nurs Health. 2002;25(3):176-188.

21. Bodnar TW, Fowler KE, Saint S. Does the structure of inpatient rounds affect medical student education? Int J Med Educ. 2013;4:96-100.

22. U.S. Department of Veterans Affairs, Office of Academic Affiliations. Medical and Dental Education Program. Available at: http://www.va. gov/oaa/GME_default.asp. Published 2012. Accessed May 08, 2013.

23. Brotherton SE, Etzel SI. Graduate medical education, 2011-2012. JAMA. 2012;308(21):2264-2279.

24. Meltzer D, Manning WG, Morrison J, et al. Effects of physician experience on costs and outcomes on an academic general medicine service: results of a trial of hospitalists. Ann Intern Med. 2002;137(11): 866-874.

25. Auerbach AD, Wachter RM, Katz P, Showstack J, Baron RB, Goldman L. Implementation of a voluntary hospitalist service at a community teaching hospital: improved clinical efficiency and patient outcomes. Ann Intern Med. 2002;137(11):859-865.

26. Sutcliffe KM, Lewton E, Rosenthal MM. Communication failures: an insidious contributor to medical mishaps. Acad Med. 2004;79(2): 186-194.

27. Weinberg DB, Miner DC, Rivlin L. 'It depends': medical residents' perspectives on working with nurses. Am J Nurs. 2009;109(7):34-44.

28. Morrison EW, Milliken FJ. Organizational silence: a barrier to change and development in a pluralistic world. Acad Manage Rev. 2000; 25(4):706-725

29. Nembhard IM, Edmondson AC. Making it safe: the effects of leader inclusiveness and professional status on psychological safety and improvement efforts in health care teams. J Organiz Behav. 2006;27: 941-966.

30. Gotlib Conn L, Reeves S, Dainty K, Kenaszchuk C, Zwarenstein M. Interprofessional communication with hospitalist and consultant physicians in general internal medicine: a qualitative study. $B M C$ Health Serv Res. 2012;12:437.

31. Chesluk BJ, Bernabeo E, Hess B, Lynn LA, Reddy S, Holmboe ES. A new tool to give hospitalists feedback to improve interprofessional teamwork and advance patient care. Health Aff (Millwood). 2012; 31(11):2485-2492.

32. Guarino CM, Ko CY, Baker LC, Klein DJ, Quiter ES, Escarce JJ. Impact of instructional practices on student satisfaction with attendings' teaching in the inpatient component of internal medicine clerkships. J Gen Intern Med. 2006;21(1):7-12.

33. Elnicki DM, Cooper A. Medical students' perceptions of the elements of effective inpatient teaching by attending physicians and housestaff. J Gen Intern Med. 2005;20(7):635-639.

34. National Board of Medical Examiners Subject Examination Program. Internal Medicine Advanced Clinical Examination, score interpretation guide. Available at: http://www.nbme.org/PDF/SampleScoreReports/Internal_Medicine_ACE_Score_Report.pdf. Published 2011. Accessed September 13, 2013.

35. Goldman L. The impact of hospitalists on medical education and the academic health system. Ann Intern Med. 1999;130(4 part 2):364-367. 